\title{
An alternating iterative algorithm for the Cauchy problem associated to the Helmholtz equation
}

\author{
L. Marin ${ }^{\text {a,* }}$, L. Elliott ${ }^{\text {b }}$, P.J. Heggs ${ }^{\text {c }}$, D.B. Ingham ${ }^{\text {b }}$, D. Lesnic ${ }^{\text {b }}$, X. Wen ${ }^{a}$

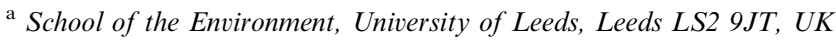 \\ ${ }^{\mathrm{b}}$ Department of Applied Mathematics, University of Leeds, Leeds LS2 9JT, UK \\ ${ }^{\mathrm{c}}$ Department of Chemical Engineering, UMIST, P.O. Box 88, Manchester M60 1QD, UK
}

Received 20 June 2002

\begin{abstract}
In this paper, the iterative algorithm proposed by Kozlov et al. [Comput. Maths. Math. Phys. 31 (1991) 45] for obtaining approximate solutions to the ill-posed Cauchy problem for the Helmholtz equation is analysed. The technique is then numerically implemented using the boundary element method (BEM). The numerical results confirm that the iterative BEM produces a convergent and stable numerical solution with respect to increasing the number of boundary elements and decreasing the amount of noise added into the input data. An efficient stopping regularising criterion is also proposed.
\end{abstract}

(c) 2002 Published by Elsevier Science B.V.

Keywords: Iterative BEM; Cauchy problem; Helmholtz equation; Inverse problem

\section{Introduction}

The Helmholtz equation arises naturally in many physical applications related to wave propagation and vibration phenomena. It is often used to describe the vibration of a structure [1], the acoustic cavity problem [2], the radiation wave [3] and the scattering of a wave [4]. Another important application of the Helmholtz equation is the problem of heat conduction in fins, see e.g. [5-7], and we focus on the latter problem in this study.

The knowledge of the Dirichlet, Neumann or mixed boundary conditions on the entire boundary of the solution domain gives rise to direct problems for the Helmholtz equation which have been extensively studied in the literature. For example, Niwa et al. [8] have studied the solution to the Helmholtz equation using the complex valued boundary element method (BEM). De Mey [9] has proposed a simplified formulation which uses the real part of the complex valued fundamental solution to construct the real part BEM for the Helmholtz equation. Whilst Hutchinson [10] has used the real part BEM in order to solve the

\footnotetext{
${ }^{*}$ Corresponding author.

E-mail address: liviu@env.leeds.ac.uk (L. Marin).
} 
vibration problems of a membrane. Later, other real-valued formulations have been developed, e.g. the multiple reciprocity method (MRBEM) [11-13] and the dual reciprocity method (DRBEM) [14-16].

The well-posedness of the direct problems of the Helmholtz equation via the removal of the eigenvalues of the Laplacian operator is well established, see e.g. [17]. Unfortunately, many engineering problems do not belong to this category. In particular, the boundary conditions are often incomplete, either in the form of underspecified and overspecified boundary conditions on different parts of the boundary or the solution is prescribed at some internal points in the domain. These are inverse problems, and it is well known that they are generally ill-posed, i.e. the existence, uniqueness and stability of their solutions are not always guaranteed.

There are important studies in the literature of the Cauchy problem for the Helmholtz equation. Unlike in direct problems, the uniqueness of the Cauchy problem is guaranteed without the necessity of removing the eigenvalues for the Laplacian. However, the Cauchy problem suffers from the non-existence and instability of the solution. A BEM-based acoustic holography technique using the singular value decomposition (SVD) for the reconstruction of sound fields generated by irregularly shaped sources has been developed by Bai [18]. The vibrational velocity, sound pressure and acoustic power on the vibrating boundary comprising an enclosed space have been reconstructed by Kim and Ih [19] who have used the SVD in order to obtain the inverse solution in the least-squares sense and to express the acoustic modal expansion between the measurement and source field. Wang and $\mathrm{Wu}$ [20] have developed a method employing the spherical wave expansion theory and a least-squares minimisation to reconstruct the acoustic pressure field from a vibrating object and their method has been extended to the reconstruction of acoustic pressure fields inside the cavity of a vibrating object by Wu and $\mathrm{Yu}$ [21]. Recently, DeLillo et al. [22] have detected the source of acoustical noise inside the cabin of a midsize aircraft from measurements of the acoustical pressure field inside the cabin by solving a linear Fredholm integral equation of the first kind. At this stage, it is worth mentioning that in the above papers the application of traditional methods, such as the Tikhonov regularisation or the truncated SVD, can solve Cauchy problems which may not have a solution and this seems redundant.

Therefore, we have decided in this study to use an iterative BEM algorithm for the solution of a Cauchy problem of a Helmholtz-type equation based on an alternating iterative procedure which consists of obtaining successive solutions to well-posed mixed boundary value problems, similar to that proposed by Kozlov et al. [23]. The strength of this iterative algorithm is that it is convergent if and only if the solution of the Cauchy problem exists, which overcomes the previous mathematical redundancy. Whilst Kozlov et al. [23] proved the mathematical convergence of the algorithm without actually finding the solution, the aim of this paper is to show the numerical stability and convergence of the present algorithm. Furthermore, in order to cease the iterative procedure before the effects of the accumulation of noise become dominant, and the errors in the numerical solution start increasing, a discrepancy stopping criterion is also proposed.

\section{Mathematical formulation}

Referring to heat transfer for the sake of the physical explanation, we assume that the temperature field $T(\underline{x})$ satisfies the Helmholtz equation in an open bounded domain $\Omega \subset \mathbb{R}^{d}$, where $d$ is the dimension of the space in which the problem is posed, usually $d \in\{1,2,3\}$, namely

$$
L T(\underline{x}) \equiv\left(\Delta+k^{2}\right) T(\underline{x})=0, \quad x \in \Omega,
$$

where $k=\alpha+\mathrm{i} \beta \in \mathbb{C}, \mathrm{i}=\sqrt{-1}$. For example, when $\alpha=0.0$ and $\beta \in \mathbb{R}$, the partial differential equation (1) models the heat conduction in a fin, see e.g. [5-7], where $T$ is the dimensionless local fin temperature, $\beta^{2}=h /(\widetilde{k} t), h$ is the surface heat transfer coefficient $\left(\mathrm{W} /\left(\mathrm{m}^{2} \mathrm{~K}\right)\right), \widetilde{k}$ is the thermal conductivity of the 
fin $(\mathrm{W} /(\mathrm{m} \mathrm{K}))$ and $t$ is the half-fin thickness $(\mathrm{m})$. We now let $n(\underline{x})$ be the outward normal vector at the boundary $\Gamma=\partial \Omega$ and $\Phi(\underline{x})$ be the flux at a point $\underline{x} \in \Gamma$ defined by

$$
\Phi(\underline{x})=\frac{\partial T}{\partial n}(\underline{x}), \quad \underline{x} \in \Gamma .
$$

The Cauchy problem under investigation requires solving the partial differential equation (1) subject to the boundary conditions

$$
T(\underline{x})=\widetilde{T}(\underline{x}), \quad \Phi(\underline{x})=\widetilde{\Phi}(\underline{x}), \quad \underline{x} \in \Gamma_{2},
$$

where $\widetilde{T}$ and $\widetilde{\Phi}$ are prescribed functions and $\Gamma_{2} \subset \Gamma$, meas $\left(\Gamma_{2}\right)>0$. In the above formulation of the boundary conditions (3), it can be seen that the boundary $\Gamma_{2}$ is overspecified by prescribing both the temperature $\left.T\right|_{\Gamma_{2}}$ and the flux $\left.\Phi\right|_{\Gamma_{2}}$, whilst the boundary $\Gamma_{1}=\Gamma \backslash \Gamma_{2}$ is underspecified since both the temperature $\left.T\right|_{\Gamma_{1}}$ and the flux $\left.\Phi\right|_{\Gamma_{1}}$ are unknown and have to be determined.

\section{Description of the algorithm}

The Cauchy problem (1) and (3) cannot be solved numerically by using a direct approach, such as the Gauss elimination method, since this problem is ill-posed. Instead we use a convergent iterative algorithm which was proposed by Kozlov et al. [23] for Cauchy problems associated to linear, elliptic, self-adjoint and positive-definite operators. This algorithm consists of the following steps:

Step 1. Specify an initial approximation $\Phi^{(0)}(\underline{x})$ for the flux on $\Gamma_{1}$ and solve the well-posed mixed boundary value problem

$$
\begin{cases}L T^{(1)}(\underline{x})=0, & \underline{x} \in \Omega, \\ \Phi^{(1)}(\underline{x}) \equiv \frac{\partial T^{(1)}}{\partial n}(\underline{x})=\Phi^{(0)}(\underline{x}), & \underline{x} \in \Gamma_{1}, \\ T^{(1)}(\underline{x})=\widetilde{T}(\underline{x}), & \underline{x} \in \Gamma_{2}\end{cases}
$$

in order to determine $T^{(1)}(\underline{x})$ for $\underline{x} \in \Omega$ and $T^{(1)}(\underline{x})$ for $\underline{x} \in \Gamma_{1}$.

Step 2. Having constructed the approximation $T^{(2 n-1)}(\underline{x}), n \geqslant 1$, the well-posed mixed boundary value problem

$$
\begin{cases}L T^{(2 n)}(\underline{x})=0, & \underline{x} \in \Omega, \\ T^{(2 n)}(\underline{x})=T^{(2 n-1)}(\underline{x}), & \underline{x} \in \Gamma_{1}, \\ \Phi^{(2 n)}(\underline{x}) \equiv \frac{\partial T^{(2 n)}}{\partial n}(\underline{x})=\widetilde{\Phi}(\underline{x}), & \underline{x} \in \Gamma_{2}\end{cases}
$$

is solved to determine $T^{(2 n)}(\underline{x})$ for $\underline{x} \in \Omega$ and $\Phi^{(2 n)}(\underline{x}) \equiv \partial T^{(2 n)}(\underline{x}) / \partial n$ for $\underline{x} \in \Gamma_{1}$.

Step 3. Having constructed the function $T^{(2 n)}(\underline{x}), n \geqslant 1$, the well-posed mixed boundary value problem

$$
\begin{cases}L T^{(2 n+1)}(\underline{x})=0, & \underline{x} \in \Omega, \\ \Phi^{(2 n+1)}(\underline{x}) \equiv \frac{\partial T^{(2 n+1)}}{\partial n}(\underline{x})=\Phi^{(2 n)}(\underline{x}), & \underline{x} \in \Gamma_{1}, \\ T^{(2 n+1)}(\underline{x})=\widetilde{T}(\underline{x}), & \underline{x} \in \Gamma_{2}\end{cases}
$$

is solved to determine $T^{(2 n+1)}(\underline{x})$ for $\underline{x} \in \Omega$ and $T^{(2 n+1)}(\underline{x})$ for $\underline{x} \in \Gamma_{1}$.

Step 4. Repeat Steps 2 and 3 until a prescribed stopping criterion is satisfied.

Remark. Let $H^{1}(\Omega)$ be the Sobolev space and $H^{1 / 2}(\Gamma)$ be the space of traces on $\Gamma$ corresponding to $H^{1}(\Omega)$, see e.g. [24]. We denote by $H^{1 / 2}\left(\Gamma_{j}\right)$ the space of functions from $H^{1 / 2}(\Gamma)$ that are bounded on $\Gamma_{j}$ and by $H^{1 / 2}\left(\Gamma_{j}\right)^{*}$ the dual space of $H^{1 / 2}\left(\Gamma_{j}\right)$ for $j=1,2$. Kozlov et al. [23] showed that if $\Gamma$ is smooth, $\widetilde{T} \in H^{1 / 2}\left(\Gamma_{2}\right)$, 
$\widetilde{\Phi} \in H^{1 / 2}\left(\Gamma_{2}\right)^{*}$ and $k$ is purely imaginary, i.e. $\alpha=0$, then the alternating algorithm based on Steps $1-4$ produces two sequences of approximate solutions $\left\{T^{(2 n)}(\underline{x})\right\}_{n \geqslant 1}$ and $\left\{T^{(2 n-1)}(\underline{x})\right\}_{n \geqslant 1}$ which both converge in $H^{1}(\Omega)$ to the solution $T(\underline{x})$ of the Cauchy problem (1) and (3), if it exists, for any initial guess $\Phi^{(0)} \in H^{1 / 2}\left(\Gamma_{1}\right)^{*}$. Also the same conclusion is obtained if at step 1 we specify an initial guess $T^{(0)} \in H^{1 / 2}\left(\Gamma_{1}\right)$, instead of an initial guess for the flux $\Phi^{(0)} \in H^{1 / 2}\left(\Gamma_{1}\right)^{*}$, and we modify accordingly the Steps 1-3 of the algorithm.

We note that if the initial guess $\Phi^{(0)}$ is in $H^{1 / 2}\left(\Gamma_{1}\right)^{*}$ and the boundary data $\widetilde{T}$ and $\widetilde{\Phi}$ are in $H^{1 / 2}\left(\Gamma_{2}\right)$ and $H^{1 / 2}\left(\Gamma_{2}\right)^{*}$, respectively, the problems (4)-(6) are well-posed and solvable in $H^{1}(\Omega)$, provided that $k^{2}$ is not an eigenvalue of the Laplacian operator $\Delta$, see [24]. These intermediate mixed well-posed problems (4)-(6) are solved using the BEM described in the following section.

\section{Boundary element method}

The Helmholtz equation (1) can also be formulated in integral form, see e.g. [17], as

$$
c(\underline{x}) T(\underline{x})+f_{\Gamma} \frac{\partial E(\underline{x}, \underline{y})}{\partial n(\underline{y})} T(\underline{y}) \mathrm{d} \Gamma(\underline{y})=\int_{\Gamma} E(\underline{x}, \underline{y}) \Phi(\underline{y}) \mathrm{d} \Gamma(\underline{y})
$$

for $\underline{x} \in \bar{\Omega}=\Omega \cup \Gamma$, where the first integral is taken in the sense of the Cauchy principal value, $c(\underline{x})=1$ for $\underline{x} \in \Omega$ and $c(\underline{x})=1 / 2$ for $\underline{x} \in \Gamma$ (smooth), and $E$ is the fundamental solution for the Helmholtz equation (1), which in two-dimensions is given by

$$
E(\underline{x}, \underline{y})=\frac{\mathrm{i}}{4} H_{0}^{(1)}(k r(\underline{x}, \underline{y})) .
$$

Here $r(\underline{x}, y)$ represents the distance between the load point $\underline{x}$ and the field point $y$ and $H_{0}^{(1)}$ is the Hankel function of order zero of the first kind. It should be noted that in practice the boundary integral equation (7) can rarely be solved analytically and thus a numerical approximation is required.

A BEM with constant boundary elements is used in order to solve the intermediate mixed well-posed boundary value problems resulting from the iterative method adopted, which is described in Section 3. Consequently, the boundary $\Gamma$ is approximated by $N$ straight line segments in a counterclockwise sense along with the temperature and the flux which are considered to be constant and take their values at the midpoint, i.e. the collocation point, also known as the node, of each element. More specifically, we have

$$
\begin{aligned}
& \Gamma \approx \bigcup_{n=1}^{N} \Gamma_{n}, \quad \Gamma_{n}=\left[\underline{y}^{n-1}, \underline{y}^{n}\right], \quad n=1, \ldots, N, \\
& \underline{y}^{N}=\underline{y}^{0}, \quad \underline{x}^{n}=\left(\underline{y}^{n-1}+\underline{y}^{n}\right) / 2, \quad n=1, \ldots, N,
\end{aligned}
$$

and

$$
T(\underline{y})=T\left(\underline{x}^{n}\right), \quad \Phi(\underline{y})=\Phi\left(\underline{x}^{n}\right), \quad \underline{y} \in \Gamma_{n}, \quad n=1, \ldots, N .
$$

By applying the boundary integral equation (7) at each collocation point $\underline{x}^{m}, m=1, \ldots, N$, and taking into account the fact that the boundary is always smooth at these points, we arrive at the following system of linear algebraic equations

$$
A \underline{T}=B \underline{\Phi}
$$

where $A$ and $B$ are matrices which depend solely on the geometry of the boundary $\Gamma$ and the vectors $\underline{T}$ and $\Phi$ consist of the discretised values of the temperature and the flux on the boundary $\Gamma$, namely 


$$
T(m)=T\left(\underline{x}^{m}\right), \quad \Phi(m)=\Phi\left(\underline{x}^{m}\right)
$$

for $m=1, \ldots, N$, and

$$
A(n, m)=\delta_{n m} / 2+f_{\Gamma_{n}} \frac{\partial E\left(\underline{x}^{m}, \underline{y}\right)}{\partial n(\underline{y})} \mathrm{d} \Gamma(\underline{y}), \quad B(n, m)=\int_{\Gamma_{n}} E\left(\underline{x}^{m}, \underline{y}\right) \mathrm{d} \Gamma(\underline{y})
$$

for $\underline{x}^{m} \in \widetilde{\Gamma}$ and $m, n=1, \ldots, N$, where $\delta_{n m}$ is the Kronecker tensor. We note that the sense of the Cauchy principal value assigned to the first integral in the boundary integral equation (7) has meaning only when $\underline{x}^{m} \in \Gamma_{n}$, as in the other cases the integral is non-singular.

If the boundaries $\Gamma_{1}$ and $\Gamma_{2}$ are discretised into $N_{1}$ and $N_{2}$ boundary elements, respectively, such that $N_{1}+N_{2}=N$, then Eq. (11) represents a system of $N$ linear algebraic equations with $2 N$ unknowns. The discretisation of the boundary conditions (3) provides the values of $2 N_{2}$ of the unknowns and the problem reduces to solving a system of $N$ equations with $2 N_{1}$ unknowns which can be generically written as

$$
C \underline{X}=\underline{F}
$$

where $F$ is computed using the boundary conditions (3), the matrix $C$ depends solely on the geometry of the boundary $\Gamma$ and the vector $\underline{X}$ contains the unknown values of the temperature and the flux on the boundary $\Gamma_{1}$.

\section{Numerical results and discussion}

In this section we illustrate the numerical results obtained using the alternating boundary element algorithm proposed in Section 4 combined with the BEM described in Section 3. In addition, we investigate the convergence with respect to the mesh size discretisation and the number of iterations when the data is exact and the stability when the data is perturbed by noise.

\subsection{Examples}

In order to present the performance of the numerical method proposed, we solve the Cauchy problem for two benchmark examples in a two-dimensional smooth geometry, namely the unit disc $\Omega=$ $\left\{\underline{x}=\left(x_{1}, x_{2}\right) \mid x_{1}^{2}+x_{2}^{2}<R^{2}\right\}, \quad R=1.0$, and the annular domain $\widetilde{\Omega}=\left\{\underline{x}=\left(x_{1}, x_{2}\right) \mid R_{i}^{2}<x_{1}^{2}+x_{2}^{2}<R_{o}^{2}\right\}$, $R_{i}=0.5$ and $R_{o}=1.0$. Piecewise smooth domains are deferred to a future numerical illustration, since at this stage the condition of a smooth domain is, as yet, required by the theoretical analysis of Kozlov et al. [23]. We assume that the boundary $\Gamma$ of the solution domain is divided into two disjointed parts, namely $\Gamma_{1}=\{\underline{x} \in \Gamma \mid 0<\theta(\underline{x})<\Theta\}$ and $\Gamma_{2}=\{\underline{x} \in \Gamma \mid \Theta<\theta(\underline{x})<2 \pi\}$ in the case of Example 1, where $\theta(\underline{x})$ is the polar angle of $\underline{x}$ and $\Theta$ is a specified angle in the interval $(0,2 \pi)$, and $\Gamma_{1}=\left\{\underline{x} \in \Gamma \mid x_{1}^{2}+x_{2}^{2}=R_{i}^{2}\right\}$ and $\Gamma_{2}=\left\{\underline{x} \in \Gamma \mid x_{1}^{2}+x_{2}^{2}=R_{o}^{2}\right\}$ in the case of Example 2. In order to illustrate the typical numerical results we have taken $\Theta=\pi / 2$. We consider the following analytical solutions for the temperature:

\section{Example 1 (Simply connected domain $\Omega$ ).}

$$
T^{(\mathrm{an})}(\underline{x})=\exp \left(a_{1} x_{1}+a_{2} x_{2}\right), \quad \underline{x}=\left(x_{1}, x_{2}\right) \in \Omega,
$$

where $k=\alpha+\mathrm{i} \beta, \alpha=0, \beta=1.0, a_{1}=0.5$ and $a_{2}=\sqrt{\beta^{2}-a_{1}^{2}}$, and

Example 2 (Double connected domain $\widetilde{\Omega}$ ).

$$
T^{(\mathrm{an})}(\underline{x})=\exp \left(a_{1} x_{1}+a_{2} x_{2}\right), \quad \underline{x}=\left(x_{1}, x_{2}\right) \in \widetilde{\Omega},
$$

where $k=\alpha+\mathrm{i} \beta, \alpha=0, \beta=2.0, a_{1}=1.0$ and $a_{2}=-\sqrt{\beta^{2}-a_{1}^{2}}$. 
These examples have a flux on the boundary $\Gamma$ given by

$$
\Phi^{(\mathrm{an})}(\underline{x})=\left[a_{1} n_{1}(\underline{x})+a_{2} n_{2}(\underline{x})\right] T^{(\mathrm{an})}(\underline{x}), \quad \underline{x}=\left(x_{1}, x_{2}\right) \in \Gamma .
$$

The Cauchy problems given by Eqs. (1) and (3) for the aforementioned examples have been solved iteratively using the BEM to provide simultaneously the unspecified boundary temperature and flux on $\Gamma_{1}$ and the temperature inside the solution domain. The number of constant boundary elements used for discretising the boundary $\Gamma$ was taken to be $N \in\{40,80,160\}$ with $N_{1}=N_{2} / 3=N / 4$ and $N_{1}=N_{2}=N / 2$ for Examples 1 and 2, respectively.

\subsection{Direct approach}

A good a priori insight into the ill-conditioning of the system of linear equations (14) is given by the condition number of the sensitivity matrix $C$, namely

$$
\text { cond } C=\operatorname{det}\left(C^{\text {tr }} C\right) \text {. }
$$

For the test examples considered, the condition numbers of the sensitivity matrix $C$ were calculated using the NAG subroutine F03AAF which computes the determinant of a matrix using Crout factorisation with partial pivoting. The condition numbers (18) for $N=20$ boundary elements corresponding to the Examples 1 and 2 are $\mathrm{O}\left(10^{-154}\right)$ and $\mathrm{O}\left(10^{-216}\right)$, respectively. When the number of boundary elements exceeds $N=20$ then the condition numbers are even smaller and the value of the determinant (18) is too small to be stored in the computer. Therefore, a direct approach to the problem produces a highly unstable solution and this is a reason why other methods, such as the proposed iterative method, have to be employed.

\subsection{Initial guess}

An arbitrary function $\Phi^{(0)} \in H^{1 / 2}\left(\Gamma_{1}\right)^{*}$ may be specified as an initial guess for the flux on $\Gamma_{1}$, but in order to improve the rate of convergence of the iterative procedure we have chosen a function which ensures the continuity of the flux at the endpoints of $\Gamma_{1}$ and which is also linear with respect to the polar angle $\theta$. For Example 1, this initial guess is given by

$$
\Phi^{(0)}(\underline{x})=\left(\frac{2 \pi-\theta(\underline{x})}{2 \pi-\Theta}\right) \Phi^{(\mathrm{an})}\left(\underline{x}_{1}\right)+\left(\frac{\theta(\underline{x})-\Theta}{2 \pi-\Theta}\right) \Phi^{(\mathrm{an})}\left(\underline{x}_{2}\right), \quad \underline{x} \in \Gamma_{1},
$$

where $\underline{x}_{j}, j=1,2$, are the endpoints of $\Gamma_{1}$, and this choice also ensures that the initial guess is not too close to the exact values $\Phi^{(\mathrm{an})}\left(x_{1}, x_{2}\right)$. For Example 2, the initial guess has been chosen as

$$
\Phi^{(0)}(\underline{x})=0, \quad \underline{x} \in \Gamma_{1} .
$$

\subsection{Convergence of the algorithm}

In order to investigate the convergence of the algorithm, at every iteration we evaluate the accuracy errors defined by

$$
e_{T}=\left\|T^{(n)}-T^{(\mathrm{an})}\right\|_{L^{2}\left(\Gamma_{1}\right)}, \quad e_{\Phi}=\left\|\Phi^{(n)}-\Phi^{(\mathrm{an})}\right\|_{L^{2}\left(\Gamma_{1}\right)},
$$

where $T^{(n)}$ and $\Phi^{(n)}$ are the temperature and the flux on the boundary $\Gamma_{1}$ retrieved after $n$ iterations, respectively, and each iteration consists of solving the two mixed well-posed problems (5) and (6). The error 

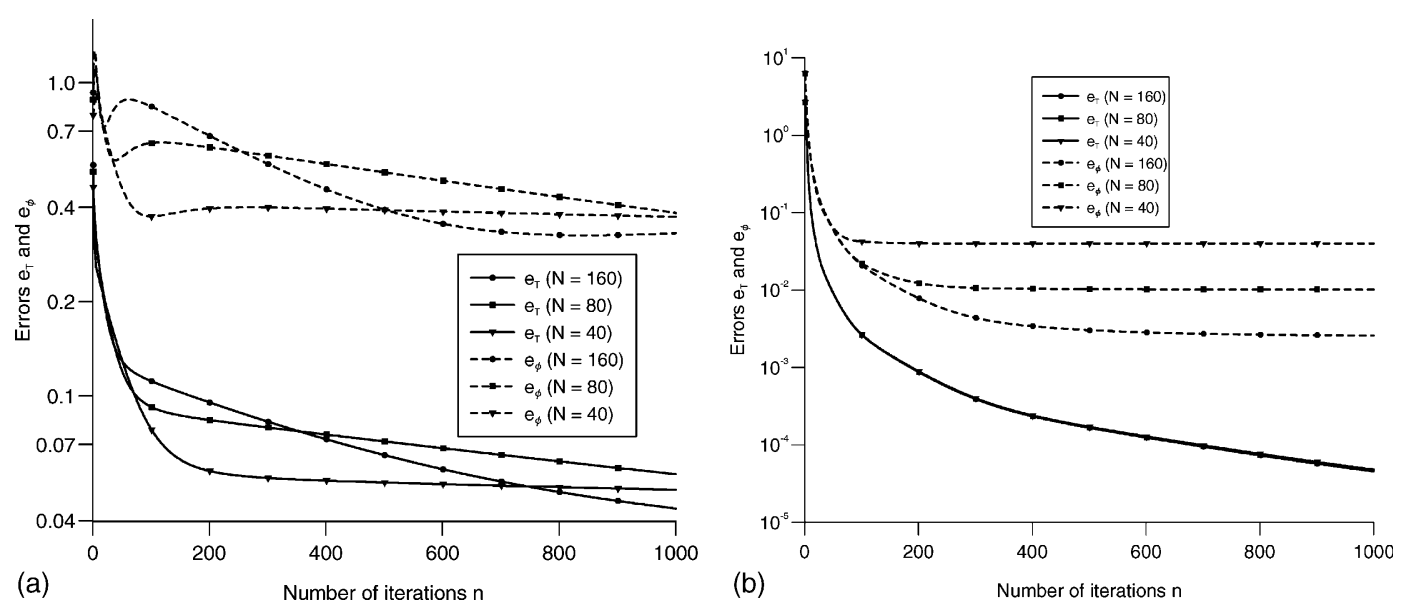

Fig. 1. The accuracy errors $e_{T}=\left\|T^{(n)}-T^{(\mathrm{an})}\right\|_{L^{2}\left(\Gamma_{1}\right)}(-)$ and $e_{\Phi}=\left\|\Phi^{(n)}-\Phi^{(\mathrm{an})}\right\|_{L^{2}\left(\Gamma_{1}\right)}(---)$ as functions of the number of iterations, $n$, obtained with $N=40(\boldsymbol{\nabla}), N=80(\mathbf{\square})$, and $N=160(\bullet)$ constant boundary elements, for (a) Example 1, and (b) Example 2.

in predicting the temperature inside the solution domain $\Omega$ or $\widetilde{\Omega}$ may also be evaluated by using the expressions

$$
e_{\Omega}=\left\|T^{(n)}-T^{(\mathrm{an})}\right\|_{L^{2}(\Omega)}, \quad e_{\widetilde{\Omega}}=\left\|T^{(n)}-T^{(\mathrm{an})}\right\|_{L^{2}(\widetilde{\Omega})} .
$$

However, this is not pursued here since both have evolutions similar to those of the errors $e_{T}$ and $e_{\Phi}$, as at each iteration the values of the temperature inside the solution domain are retrieved from the values of the temperature $T$ and the flux $\Phi$ on the boundary $\Gamma$.

Fig. 1(a) and (b) shows the accuracy errors $e_{T}$ and $e_{\Phi}$ as functions of the number of iterations, $n$, obtained for the Cauchy problems given by Examples 1 and 2, respectively, for $N \in\{40,80,160\}$ when using "exact boundary data" for the inverse problem, i.e. boundary data obtained by solving a direct well-posed problem, namely

$$
\begin{cases}L T(\underline{x})=0, & \underline{x} \in \Omega, \\ \Phi(\underline{x}) \equiv \frac{\partial T}{\partial n}(\underline{x})=\Phi^{(\mathrm{an})}(\underline{x}), & \underline{x} \in \Gamma_{1}, \\ T(\underline{x})=T^{(\mathrm{an})}(\underline{x}), & \underline{x} \in \Gamma_{2} .\end{cases}
$$

It can be seen from these figures that both errors $e_{T}$ and $e_{\Phi}$ keep decreasing, even after a large number of iterations, e.g. $N=1000$, and, as expected, $e_{T}<e_{\Phi}$, i.e. temperatures are more accurate than fluxes. Furthermore, as $N$ increases, the errors $e_{T}$ and $e_{\Phi}$ decrease showing that for Example $1, N \geqslant 160$, and for Example 2, $N \geqslant 40$, ensure a sufficient discretisation for the accuracy to be achieved. From Fig. 1(a) and (b), it can be concluded that the alternating iterative algorithm described in Section 3 is convergent with respect to increasing the number of iterations, provided that exact boundary data is prescribed.

Although not illustrated here, an important conclusion is reported, namely, that the alternating iterative algorithm described in Section 3 is not convergent for the differential operator $L \equiv \Delta+k^{2}$ for $k=\alpha+\mathrm{i} \beta$, $\alpha \in \mathbb{R}$ and $\beta=0$, both errors $e_{T}$ and $e_{\Phi}$ defined by relations (21) "blow up" after the first iteration. The reason is that the proof of convergence of the iterative algorithm of Kozlov et al. [23] requires, as a necessary condition, $L \equiv \Delta+k^{2}$ to be positive-definite differential operators and this is not the case when $k$ is real.

The numerical solutions for the temperature $\left.T\right|_{\Gamma_{1}}$ obtained after $n=1000$ iterations for the Cauchy problems given by Examples 1 and 2 are presented in Fig. 2(a) and (b), respectively. From these figures, it can be seen that the accuracy in predicting the temperature distribution on the boundary $\Gamma_{1}$ is reasonable 

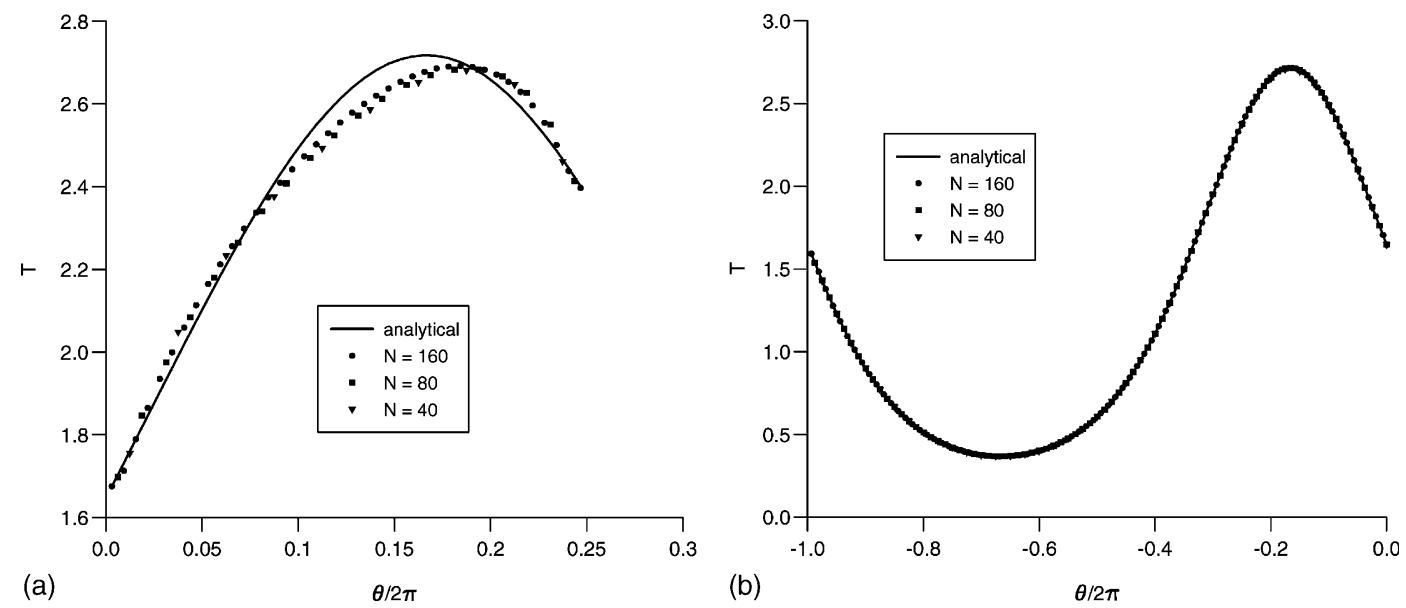

Fig. 2. The analytical solution $T^{(\mathrm{an})}(-)$ and the numerical solution $T^{(\text {num })}$ obtained with $N=40(\boldsymbol{\nabla}), N=80(\boldsymbol{\square})$, and $N=160$ constant boundary elements, on the underspecified boundary $\Gamma_{1}$, for (a) Example 1, and (b) Example 2.

for Example 1 and very good for Example 2. The behaviour of the numerical solution for the flux $\left.\Phi\right|_{\Gamma_{1}}$, along with the analytical flux solution on the boundary $\Gamma_{1}$, after $n=1000$ iterations for the Cauchy problems considered in Examples 1 and 2 is presented in Fig. 3(a) and (b), respectively. As expected, the errors in predicting the flux $\left.\Phi\right|_{\Gamma_{1}}$ are larger than the errors in predicting the temperature $\left.T\right|_{\Gamma_{1}}$ since the flux contains higher-order derivatives. Moreover, from Figs. 2 and 3 it can be seen that the numerical solutions for the temperature $\left.T\right|_{\Gamma_{1}}$ and the flux $\left.\Phi\right|_{\Gamma_{1}}$ are more accurate for the Cauchy problem corresponding to Example 2, i.e. the solution domain is an annulus, than for the Cauchy problem given by Example 1, i.e. the solution domain is a disk. The reason for this is that $\bar{\Gamma}_{1} \cap \bar{\Gamma}_{2}=\emptyset$ in the case of Example 2, whilst $\bar{\Gamma}_{1} \cap \bar{\Gamma}_{2} \neq \emptyset$ in the case of Example 1, i.e. there exist two points where the constant BEM changes to mixed boundary conditions. It is well known, see e.g. [25] and Schiavone [26], that the gradient of the temperature $T$ possesses singularities at the points where the data changes from temperature boundary conditions to flux

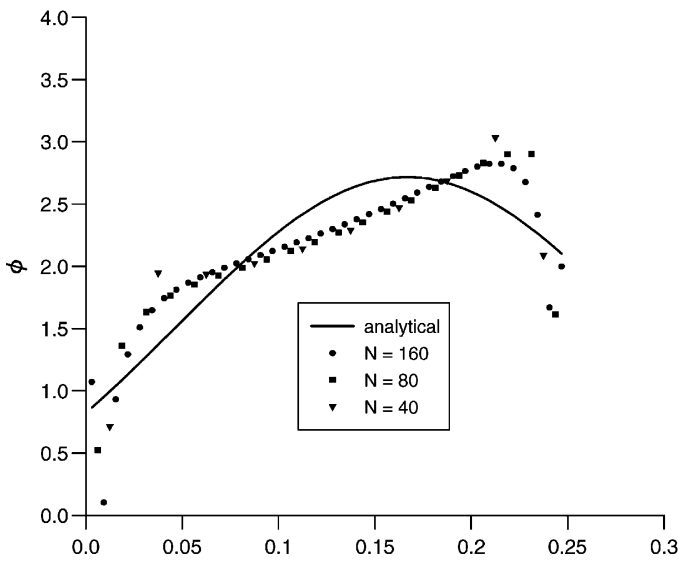

(a)

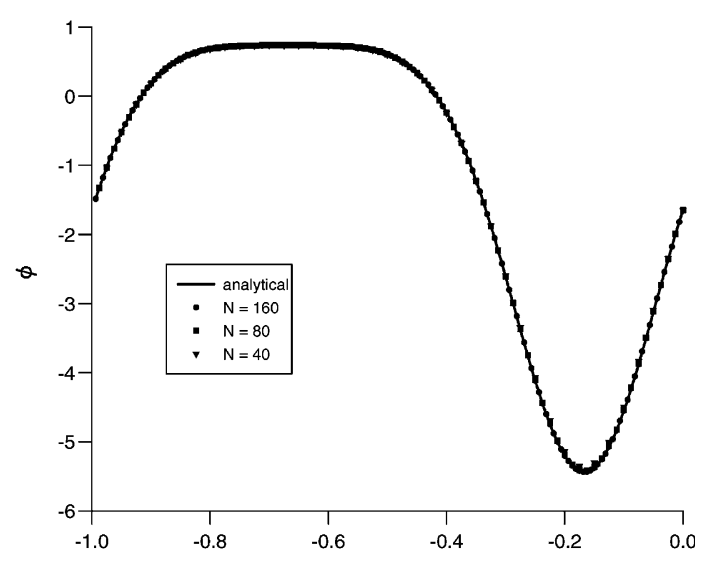

(b) $\theta / 2 \pi$

Fig. 3. The analytical solution $\Phi^{(\mathrm{an})}(-)$ and the numerical solution $\Phi^{(\mathrm{num})}$ obtained with $N=40(\boldsymbol{\nabla}), N=80(\boldsymbol{\square})$, and $N=160$ constant boundary elements, on the underspecified boundary $\Gamma_{1}$, for (a) Example 1, and (b) Example 2. 

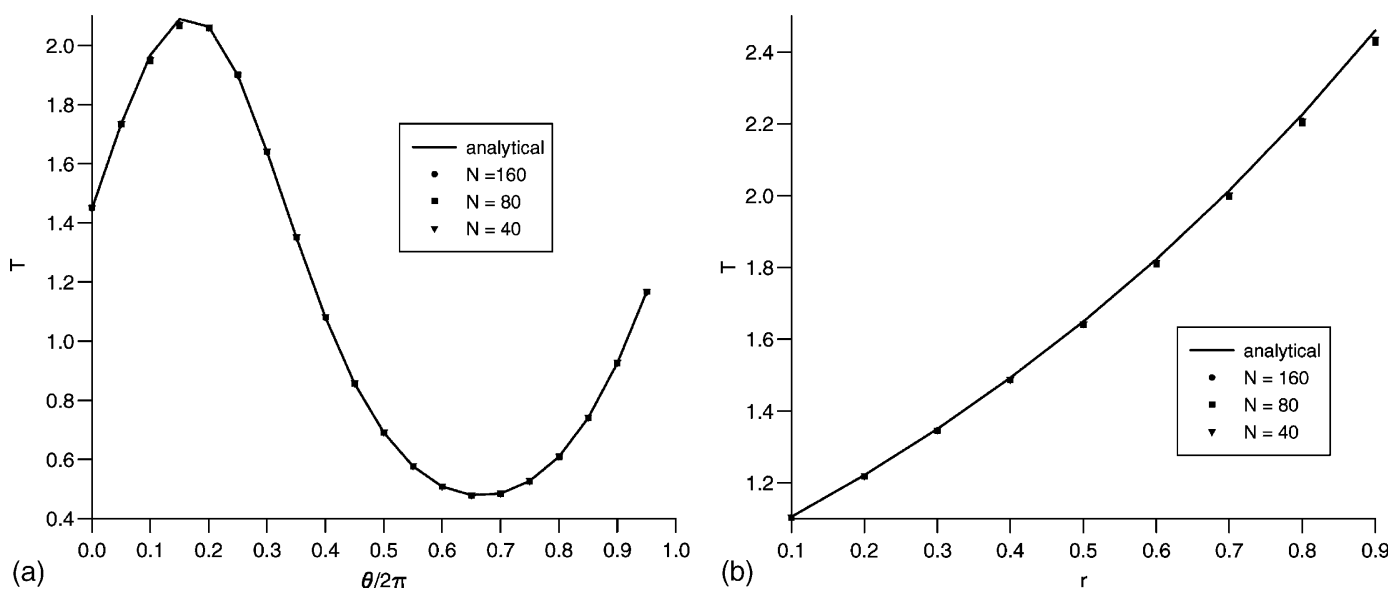

Fig. 4. The analytical solution $T^{(\text {an })}(-)$ and the numerical solution $T^{(\text {num })}$ obtained with $N=40(\boldsymbol{\nabla}), N=80(\boldsymbol{\square})$, and $N=160(\boldsymbol{\bullet})$ constant boundary elements, at the internal points (a) $\underline{x} \in \Gamma_{r}$, and (b) $\underline{x} \in \Gamma_{\theta}$, for Example 1 .

boundary conditions, even if the temperature and the flux data are of class $\mathscr{C}^{\infty}$. Consequently, the classical solution for the temperature $T$ cannot be smooth, although its smoothness can be improved if the temperature and the flux data are required to satisfy an increasing number (increasing with smoothness) of additional conditions, see also [27]. Nevertheless, in the numerical implementation one may use linear boundary elements to enforce a smooth temperature across the junctions $\bar{\Gamma}_{1} \cap \bar{\Gamma}_{2}$ or, even better, weighted functions at each iteration of the algorithm in order to cancel the singularity, see [28], but this will be investigated in a future work.

In Fig. 4(a) and (b) we present the numerical solution for the temperature $T$ obtained after $n=1000$ iterations and using various numbers $N \in\{40,80,160\}$ of boundary elements in comparison with its analytical value at the internal points $\underline{x}$ located on an interior circle, $\Gamma_{r}=\left\{\underline{x}=\left(x_{1}, x_{2}\right) \mid r(\underline{x})=3 / 4\right\}$, and along a radius, $\Gamma_{\theta}=\left\{\underline{x}=\left(x_{1}, x_{2}\right) \mid \theta(\underline{x})=\pi / 3\right\}$, respectively, for the Cauchy problem considered in Example 1 . From these figures it can be seen that the numerical temperature retrieved at the internal points considered represents a very good approximation of its exact value and it converges to the analytical solution within $N=40$ boundary elements. Similar results have been obtained for the Cauchy problem corresponding to Example 2 and hence they are not presented here.

For the Cauchy problem investigated in this paper it was found that the proposed iterative BEM algorithm produces an accurate and convergent numerical solution for both the missing boundary temperature and flux, as well as the temperature inside the solution domain, with respect to increasing the number of iterations, $n$, and the number of boundary elements, $N$, provided that exact input data is used. However, exact data is seldom available in practice since measurement errors always include noise in the prescribed boundary conditions and this is investigated next.

\subsection{Stopping criterion}

Once the convergence with respect to increasing $N$ of the numerical solution to the exact solution has been established, we fix $N=40$ and investigate the stability of the numerical solution for Example 2 only. Fig. 5 presents the accuracy errors $e_{T}$ and $e_{\Phi}$ for various levels of Gaussian random noise $p \in\{1,2\} \%$ added into the temperature data $\left.\widetilde{T}\right|_{\Gamma_{2}}$. From this figure it can be seen that as $p$ decreases then $e_{T}$ and $e_{\Phi}$ decrease. However, the errors in predicting the temperature and the flux on the underspecified boundary $\Gamma_{1}$ decrease up to a certain iteration number and after that they start increasing. If the iterative process is 


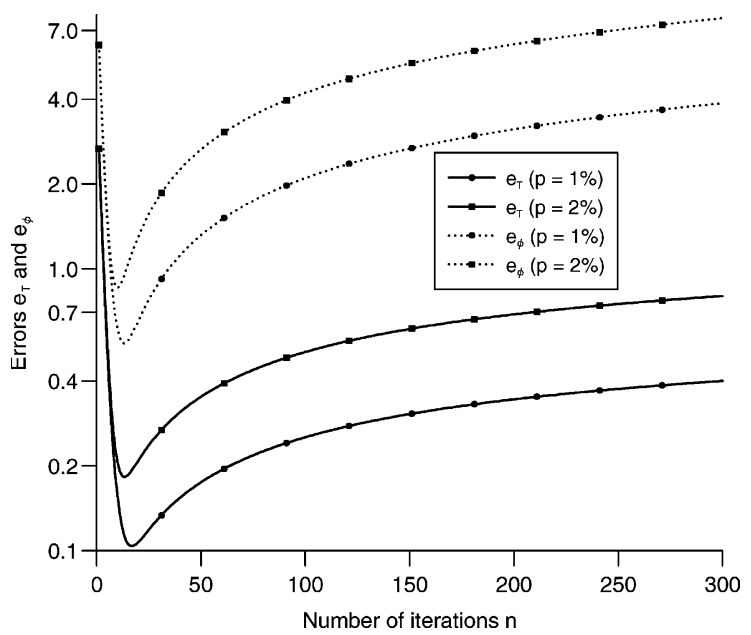

Fig. 5. The accuracy errors $e_{T}=\left\|T^{(n)}-T^{(\text {an })}\right\|_{L^{2}\left(\Gamma_{1}\right)}(-)$ and $e_{\Phi}=\left\|\Phi^{(n)}-\Phi^{(\mathrm{an})}\right\|_{L^{2}\left(\Gamma_{1}\right)}(---)$ as functions of the number of iterations, $n$, obtained with $N=40$ constant boundary elements and several amounts of noise $p=1 \%(\bullet)$ and $p=2 \%(\boldsymbol{\square})$ added into the input data $\left.\widetilde{T}\right|_{\Gamma_{2}}$, for Example 2 .

continued beyond this point then the numerical solutions lose their smoothness and become highly oscillatory and unbounded, i.e. unstable. Therefore, a regularising stopping criterion must be used in order to terminate the iterative process at the point where the errors in the numerical solutions start increasing.

If we evaluate the Euclidean norm of the vector $C \underline{X}-\underline{F}$ then this should tend to zero as $\underline{X}$ tends to the exact solution. Hence after each iteration we evaluate the error

$$
E=\left\|C \underline{X}^{(n)}-\underline{F}\right\|_{2},
$$

where $\underline{X}^{(n)}$ is the vector obtained from the values of the temperature and the flux on the boundary $\Gamma_{1}$ retrieved after $n$ iterations. The error $E$ includes information on both the temperature and the flux and it is expected to provide an appropriate stopping criterion. Indeed, if we investigate the error $E$ obtained at every iteration for Example 2 for various levels of Gaussian random noise added into the input temperature data $\left.\widetilde{T}\right|_{\Gamma_{2}}$, we obtain the curves graphically represented in Fig. 6.

A natural stopping criterion is given by the discrepancy principle which ceases the iterative procedure described above for

$$
n \in \mathbb{N}: \quad E=\left\|C \underline{X}^{(n)}-\underline{F}\right\|_{2} \leqslant \varepsilon,
$$

where $\varepsilon$ is a measure of the level of noise in the measurements of the data on the overdetermined boundary $\Gamma_{2}$, see [29].

As mentioned in the previous section, for exact data the iterative process is convergent with respect to increasing the number of iterations, $n$, since the accuracy errors $e_{T}$ and $e_{\Phi}$ keep decreasing even after a large number of iterations, see Fig. 1(a) and (b). It should be noted in this case that a stopping criterion is not necessary since the numerical solution is convergent with respect to increasing the number of iterations. However, even in this case the errors $E, e_{T}$ and $e_{\Phi}$ have a similar behaviour and the error $E$ may be used to stop the iterative process at the point where the rate of convergence is very small and no substantial improvement in the numerical solution is obtained even if the iterative process is continued. Therefore, it can 


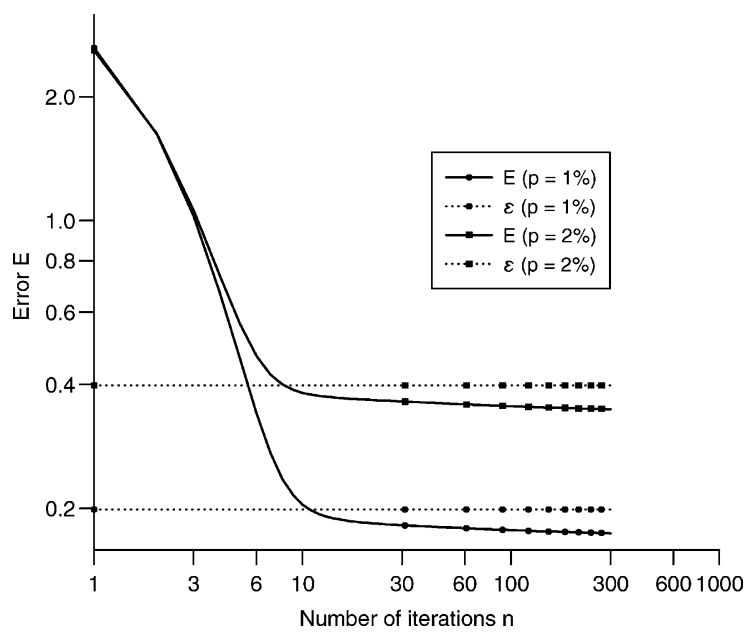

Fig. 6. The convergence error $E=\left\|C \underline{X}^{(n)}-\underline{F}\right\|_{2}(-)$ as a function of the number of iterations, $n$, and the value of $\varepsilon(\cdots)$ given by the discrepancy principle obtained with $N=40$ constant boundary elements and several amounts of noise $p=1 \%(\bullet)$ and $p=2 \%$ added into the input data $\left.\widetilde{T}\right|_{\Gamma_{2}}$, for Example 2 .

be concluded that the regularising stopping criterion proposed is very efficient in locating the point where the errors start increasing and the iterative process should be ceased.

\subsection{Stability of the algorithm}

Based on the stopping criterion described in Section 5.5, the numerical results obtained for the temperature $T$ and the flux $\Phi$ on the boundary $\Gamma_{1}$ are presented in Figs. 7 and 8, respectively, for various levels of noise added into the temperature data on the boundary $\Gamma_{2}$. From these figures it can be seen that the

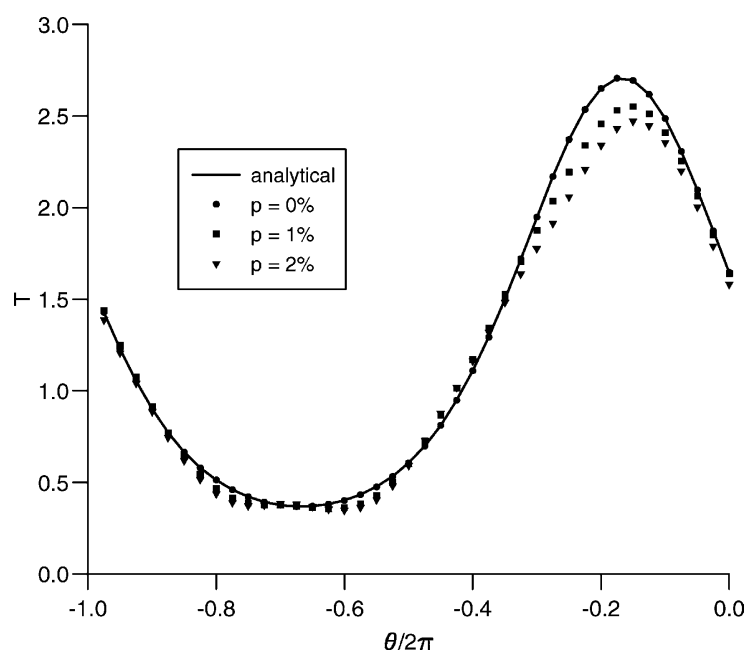

Fig. 7. The analytical solution $T^{(\text {an })}(-)$ and the numerical solution $T^{(\text {num })}$ obtained with $N=40$ constant boundary elements and several amounts of noise $p=0 \%(\boldsymbol{\bullet}), p=1 \%(\boldsymbol{\square})$, and $p=2 \%(\boldsymbol{\nabla})$ added into the input data $\left.\widetilde{T}\right|_{\Gamma_{2}}$, for Example 2 . 


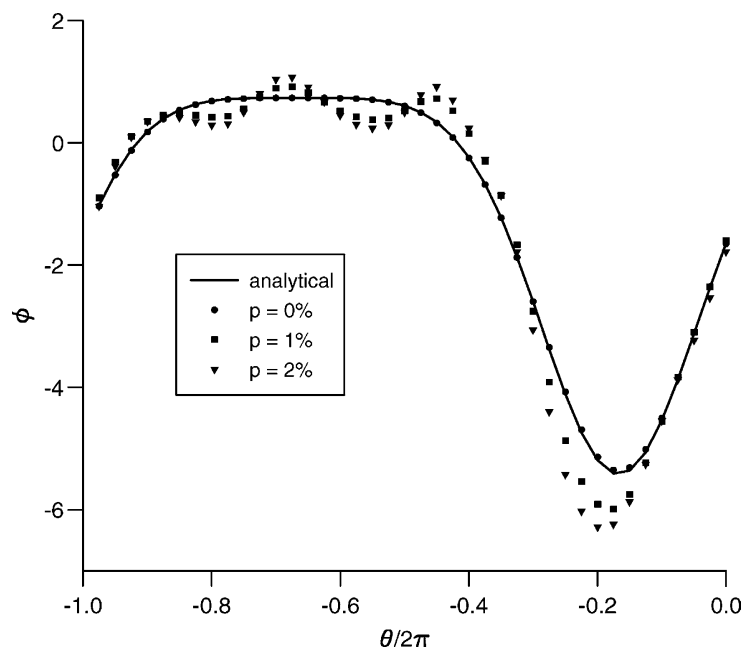

Fig. 8. The analytical solution $\Phi^{(\mathrm{an})}(-)$ and the numerical solution $\Phi^{(\mathrm{num})}$ obtained with $N=40$ constant boundary elements and several amounts of noise $p=0 \%(\bullet), p=1 \%(\boldsymbol{\square})$, and $p=2 \%(\boldsymbol{\nabla})$ added into the input data $\left.\widetilde{T}\right|_{\Gamma_{2}}$, for Example 2 .

Table 1

The errors $e_{T}$ and $e_{\Phi}$ and the value of $\varepsilon$ given by the discrepancy principle obtained with $N=40$ boundary elements and various amounts of noise added into the input data $\left.T\right|_{\Gamma_{1}}$, namely $p \in\{0,1,2\}$, for Example 2

\begin{tabular}{llll}
\hline Error & $p=0 \%$ & $p=1 \%$ & $p=2 \%$ \\
\hline$e_{T}$ & $4.02 \times 10^{-4}$ & $1.35 \times 10^{-1}$ & $2.27 \times 10^{-1}$ \\
$e_{\Phi}$ & $3.99 \times 10^{-2}$ & $5.71 \times 10^{-1}$ & $8.70 \times 10^{-1}$ \\
$\varepsilon$ & 0.00 & $1.99 \times 10^{-1}$ & $3.98 \times 10^{-1}$ \\
\hline
\end{tabular}

numerical solution is a stable approximation to the exact solution, free of unbounded and rapid oscillations. The same conclusion can be drawn from Table 1 which presents the errors $e_{T}$ and $e_{\Phi}$ obtained for the Cauchy problem given by Example 2 for various levels of noise added into the input temperature data. From this table, as well as from Figs. 7 and 8 , it should also be noted that the numerical solution converges to the exact solution as the level of noise, $p$, added into the input boundary data decreases.

From the numerical results presented in this section, it can be concluded that the stopping criterion developed in Section 5.5 has a regularising effect and the numerical solution obtained by the iterative BEM described in this paper is convergent and stable with respect to increasing the mesh size discretisation and decreasing the level of noise added into the input data, respectively.

\section{Conclusions}

In this paper we have investigated the Cauchy problem for the Helmholtz equation in the two-dimensional case. In order to deal with the instabilities of the solution of this ill-posed problem, an iterative BEM was employed which reduced the Cauchy problem to solving a sequence of well-posed boundary value problems. A stopping criterion, necessary for ceasing the iterations at the point where the accumulation of noise becomes dominant and the errors in predicting the exact solution increase, has also been presented. 
The numerical results obtained for various numbers of boundary elements and various amounts of noise added to the input data showed that the BEM produces a convergent, stable and consistent numerical solution with respect to increasing the number of boundary elements and decreasing the amount of noise.

\section{Acknowledgement}

L. Marin would like to acknowledge the financial support received from the EPSRC.

\section{References}

[1] D.E. Beskos, Boundary element method in dynamic analysis: Part II (1986-1996), ASME Appl. Mech. Rev. 50 (1997) $149-197$.

[2] J.T. Chen, F.C. Wong, Dual formulation of multiple reciprocity method for the acoustic mode of a cavity with a thin partition, J. Sound Vibration 217 (1998) 75-95.

[3] I. Harari, P.E. Barbone, M. Slavutin, R. Shalom, Boundary infinite elements for the Helmholtz equation in exterior domains, Int. J. Numer. Meth. Engrg. 41 (1998) 1105-1131.

[4] W.S. Hall, X.Q. Mao, A boundary element investigation of irregular frequencies in electromagnetic scattering, Engrg. Anal. Boundary Elem. 16 (1995) 245-252.

[5] D.Q. Kern, A.D. Kraus, Extended Surface Heat Transfer, McGraw-Hill, New York, 1972.

[6] M. Manzoor, D.B. Ingham, P.J. Heggs, The one-dimensional analysis of fin assembly heat transfer, ASME J. Heat Transfer 105 (1983) 646-651.

[7] A.S. Wood, G.E. Tupholme, M.I.H. Bhatti, P.J. Heggs, Steady-state heat transfer through extended plane surfaces, Int. Comm. Heat Mass Transfer 22 (1995) 99-109.

[8] Y. Niwa, S. Kobayashi, M. Kitahara, Determination of eigenvalue by boundary element method, Chapter 7, in: P.K. Banerjee, R. Shaw (Eds.), Development in Boundary Element Methods, Applied Science Publisher, New York, 1982.

[9] G. De Mey, A simplified integral equation method for the calculation of the eigenvalues of Helmholtz equation, Int. J. Numer. Meth. Engrg. 11 (1977) 1340-1342.

[10] J.R. Hutchinson, An alternative BEM formulation applied to membrane vibrations, in: C.A. Brebbia, G. Maier (Eds.), Boundary Elements VII, Springer Verlag, Berlin, 1985, pp. 613-625.

[11] A.J. Nowak, C.A. Brebbia, Solving Helmholtz equation by boundary elements using multiple reciprocity method, in: G.M. Calomagno, C.A. Brebbia (Eds.), Computer Experiment in Fluid Flow, CMP/Springer Verlag, Berlin, 1989, pp. $265-270$.

[12] N. Kamiya, E. Andoh, Eigenvalue analysis by boundary element method, J. Sound Vibration 160 (1993) $279-287$.

[13] N. Kamiya, E. Andoh, K. Nogae, Eigenvalue analysis by boundary element method: New developments, Engrg. Anal. Boundary Elem. 16 (1993) 203-207.

[14] C.F. Nardini, C.A. Brebbia, A new approach to free vibration analysis using boundary elements, in: C.A. Brebbia, T. Futagami, M. Tanaka (Eds.), Boundary Elements V, Springer Verlag, Berlin, 1983, pp. 719-730.

[15] J.P. Agnantiaris, D. Polyzer, D. Beskos, Three-dimensional structural vibration analysis by the dual reciprocity BEM, Comput. Mech. 21 (1998) 372-381.

[16] M.A. Golberg, C.S. Chen, H. Bowman, H. Power, Some comments on the use of radial basis functions in the dual reciprocity method, Comput. Mech. 22 (1998) 61-69.

[17] G. Chen, J. Zhou, Boundary Element Methods, Academic Press, London, 1992.

[18] M.R. Bai, Application of BEM-based acoustic holography to radiation analysis of sound sources with arbitrarily shaped geometries, J. Acoust. Soc. Am. 92 (1992) 533-549.

[19] B.K. Kim, J.G. Ih, On the reconstruction of the vibro-acoustic field over the surface enclosing an interior space using the boundary element method, J. Acoust. Soc. Am. 100 (1996) 3003-3016.

[20] Z. Wang, S.R. Wu, Helmholtz equation-least-squares method for reconstructing the acoustic pressure field, J. Acoust. Soc. Am. 102 (1997) 2020-2032.

[21] S.R. Wu, J. Yu, Application of BEM-based acoustic holography to radiation analysis of sound sources with arbitrarily shaped geometries, J. Acoust. Soc. Am. 104 (1998) 2054-2060.

[22] T. DeLillo, V. Isakov, N. Valdivia, L. Wang, The detection of the source of acoustical noise in two dimensions, SIAM J. Appl. Math. 61 (2001) 2104-2121.

[23] V.A. Kozlov, V.G. Maz'ya, A.F. Fomin, An iterative method for solving the Cauchy problem for elliptic equations, Comput. Maths. Math. Phys. 31 (1991) 45-52.

[24] J.L. Lions, E. Magenes, Non-Homogeneous Boundary Value Problems and Their Applications, Springer Verlag, Berlin, 1972. 
[25] G. Fichera, Sul problema della derivata obliqua e sul problema misto per l'equazione di Laplace, Boll. Un. Mat. Ital. 7 (1952) 367-377.

[26] P. Schiavone, Mixed problem in the theory of elastic plates with transverse shear deformation, Q. Jl. Mech. Appl. Math. 50 (1997) 239-249.

[27] W.L. Wendland, E. Stephan, G.C. Hsiao, On the integral equation method for the plane mixed boundary value problem for the Laplacian, Math. Meth. Appl. Sci. 1 (1979) 265-321.

[28] T. Johansson, private communication.

[29] V.A. Morozov, On the solution of functional equations by the method of regularization, Soviet Math. Dokl. 167 (1966) $414-417$. 